\title{
Endoscopic mucosal resection of duodenal carcinoid
}

A 45-year-old man with a history of renal cell carcinoma and immunosuppression presented to our institution with melena. Upper gastrointestinal endoscopy demonstrated two nodules in the duodenal bulb. Biopsies revealed a circumscribed neoplasm in the duodenal submucosa comprising nests of epithelial cells with uniform round nuclei and abundant finely granular amphophilic cytoplasm. Chromogranin immunostaining confirmed the diagnosis of a well-differentiated neuroendocrine tumor consistent with carcinoid ( $\bullet$ Fig. 1 ).

The patient's melena resolved with no clear cause identified. He denied symptoms of carcinoid syndrome including flushing, diarrhea, facial skin lesions, and shortness of breath. A metastatic work-up was performed with no evidence of spread of disease. The metastatic work-up included an octreotide scan with possible liver uptake, subsequent magnetic resonance imaging (MRI) of the liver with no evidence of disease, and video capsule endoscopy and magnetic resonance enterography with no additional small-intestinal lesions identified. Endoscopic ultrasound (EUS) showed two 1-cm subepithelial nodules in the duodenal bulb with no evidence of invasion into the muscularis propria.

Endoscopic mucosal resection (EMR) of the duodenal bulb carcinoid lesions was performed. Both lesions were injected with a solution consisting of $500 \mathrm{~mL}$ of $6 \%$ hydroxyethyl starch (BioTime, Inc., Berkeley, California, USA), 1 mL of 1 : 10000 dilu- tion epinephrine, and indigo carmine to provide lift prior to their en bloc removal with a hot polypectomy snare (Boston Scientific, Marlborough, Massachusetts, USA). Hemostatic clips were placed at the site of both EMRs ( $\bullet$ Video 1).

Pathology of the specimen revealed a complete $\mathrm{R} 0$ resection with negative resection margins. Repeat biopsies at 3-month follow-up revealed no evidence of disease recurrence.

Duodenal carcinoids account for less than $5 \%$ of all carcinoid tumors [1,2]. The majority of patients with small-bowel carcinoid tumors present with metastases to lymph nodes or the liver, and 5\%-7\% present with carcinoid syndrome [3]. As a result, the most important consideration in determining whether patients are candidates for endoscopic treatment is evaluation for metastatic disease [4]. Carcinoid tumors with evidence of lymphatic or distant metastases should not be treated by endoscopic resection [5]. Tumor size is an unreliable predictor of metastatic disease, with metastases having been reported from tumors measuring less than $0.5 \mathrm{~cm}$ in diameter [3]. Although consensus guidelines are lacking, duodenal carcinoid tumors that are less than $1 \mathrm{~cm}$ in size and limited to the submucosa with no evidence of lymphatic or metastatic disease are candidates for EMR [6].

\section{Endoscopy_UCTN_Code_TTT_1AO_2AG}

Competing interests: Michel Kahaleh: Consultant for Boston Scientific, Xlumena

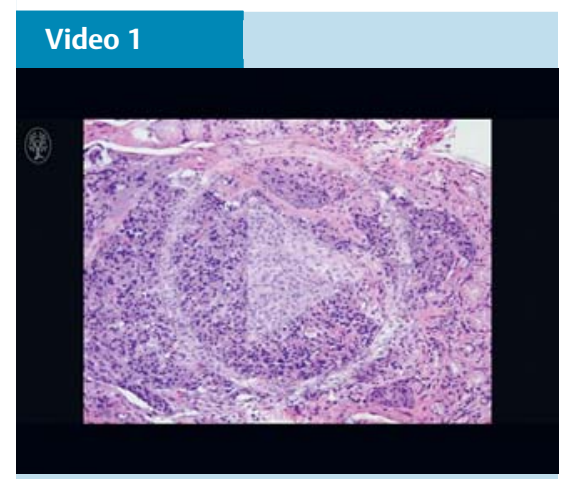

Endoscopic view of nodules at the duodenal bulb; histology of biopsies were consistent with carcinoid; endoscopic ultrasound (EUS) view with no evidence of invasion into the muscularis propria; endoscopic mucosal resection (EMR) of the duodenal carcinoid lesions.

and Concordia lab. Research for Boston Scientific, Fuji, Pentax, Gore, MI tech, Mauna Kea, EMcision, Aspire, GI Dynamics, Cook, Apollo Endosurgery, NinePoint Medical, and Merit.

Reem Sharaiha: Consultant for Apollo Endosurgery.

All other authors have no conflicts of interest.

\section{Nikhil A. Kumta, Amit Desai, Rushabh Doshi, Michel Kahaleh, Reem Z. Sharaiha}

Division of Gastroenterology and Hepatology, Weill Cornell Medical College, New York, New York, USA

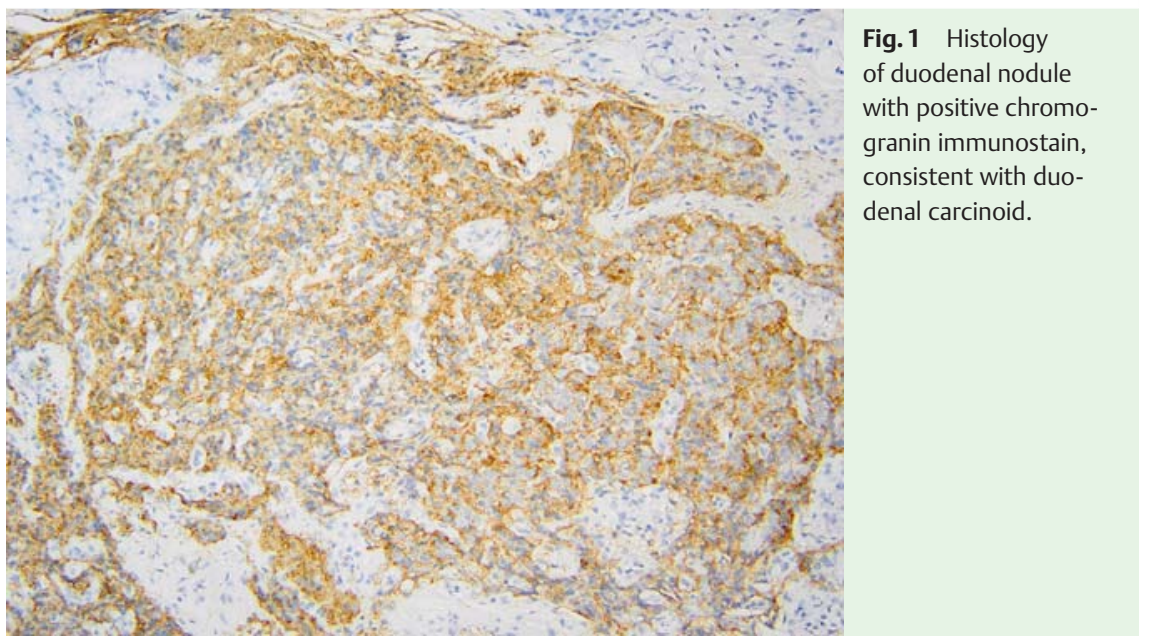




\section{References}

1 Otaki $Y$, Homma $K$, Nawata $Y$ et al. Endoscopic mucosal resection with circumferential mucosal incision of duodenal carcinoid tumors. World J Gastrointest Endosc 2013; 5: $197-200$

2 Hoffmann KM, Furukawa M, Jensen RT. Duodenal neuroendocrine tumors: Classification, functional syndromes, diagnosis and medical treatment. Best Pract Res Clin Gastroenterol 2005; 19: 675-697

3 Kulke MH, Mayer RJ. Carcinoid tumors. NEJM 1999; 340: 858-868
4 Abraham A, Singh J, Siddiqui G et al. Endoscopic management of a primary duodenal carcinoid tumor. Case Rep Gastroenterol 2012; 6: 135-142

5 Zyromski NJ, Kendrick ML, Nagorney DM et al. Duodenal carcinoid tumors: How aggressive should we be? J Gastrointest Surg 2001; 5: $588-593$

$6 \mathrm{Kim} \mathrm{GH,} \mathrm{Kim} \mathrm{JIl,Jeon} \mathrm{SW} \mathrm{et} \mathrm{al.} \mathrm{Endoscopic} \mathrm{re-}$ section for duodenal carcinoid tumors: A multicenter, retrospective study. J Gastroenterol Hepatol 2014; 29: 318-324

\section{Bibliography}

DOI http://dx.doi.org/

$10.1055 / \mathrm{s}-0042-105648$

Endoscopy 2016; 48: E158-E159

(C) Georg Thieme Verlag KG

Stuttgart · New York

ISSN 0013-726X

\section{Corresponding author}

\section{Nikhil A. Kumta, MD, MS}

Joan and Sanford I Weill Medical College of Cornell University - Gastroenterology and Hepatology

1305 York Ave 4th Floor

New York

New York 10065

United States of America

Fax: +1-646-962-4000

Nikhil.Kumta@gmail.com 